\title{
Incongruencia de género en niñas, niños y adolescentes: intervenciones transafirmativas en Hospital las Higueras Talcahuano
}

\author{
Gender incongruity in girls, boys and adolescents: \\ transaffirmative intervention at Las Higueras Hospital, Talcahuano.
}

Dorca Retamal Parra', Wladimir Hermosilla Rubio', Mónica Arancibia Cabalá2, Miguel Medel Arriagada', Catalina Retamal Vargas ${ }^{3}$

\section{Resumen:}

Introducción: incongruencia de género (IG) en niñas, niños y adolescentes (NNA), es un tema que se ha ido visibilizando cada vez más y es definida como una marcada discordancia entre la identidad de género y el sexo asignado al nacer. El objetivo es realizar una revisión de la evidencia en relación a la IG en NNA, las intervenciones recomendadas basadas en evidencia y finalmente dar a conocer la experiencia local en el Hospital las Higueras de Talcahuano. Métodos: se realizó una búsqueda en PUBMED artículos en inglés y español, desde 2011 a 2019. De los artículos encontrados, se revisó además la bibliografía de ellos, sumando a la revisión las publicaciones que fueran pertinentes. En la revisión práctica se realizó una sistematización de las intervenciones que se llevan a cabo a nivel endocrinológico y en salud mental. Resultados: los resultados indican que la prevalencia ha ido en aumento, al igual que la prevalencia hombre trans. Existen indicadores específicos para poder diferenciar la desistencia o persistencia de la IG. Las condiciones asociadas tales como ansiedad, depresión, ideación y conducta suicidas son generadas por el estrés de minorías y la intervención más apropiada es la afirmativa. Existe alta varianza de género en personas de condición trastorno de espectro autista (TEA), siendo mayor en las niñas que en los niños. Desde marzo 2019 Hospital las Higueras de Talcahuano inicia intervención transafirmativa con IG en NNA. Conclusiones: es necesario visibilizar el tema y despatologizarlo para que las(os) NNA tengan mayor acceso a apoyo transafirmativo.

Palabras clave: incongruencia de género; transgénero; niñas(os); adolescentes; intervenciones transafirmativas,

\begin{abstract}
:
Introduction: Gender incongruity (GI) in boys, girls, and adolescents (BGA), is becoming an ever more visible issue. It is defined as a marked misalignment between gender identity and the sex assigned at birth. Objective: to make a review of evidence regarding $\mathrm{GI}$ in BGA, the recommended evidence-based interventions and, ultimately, to show the local experience at Las Higueras Hospital in Talcahuano. Methods: A search of articles written in English and Spanish between 2011 and 2019 was performed in PUBMED and, from the articles found, their biblographies were also revised, adding the pertinent publications to the revision process. In the practical revision, a systematization of the interventions performed at an endocrinological level and in mental health, was also made. Results: the results indicate that prevalence has been increasing, as has male trans prevalence. There are specific indicators to differentiate $\mathrm{Gl}$ desistance or persistance. The associated conditions such as anxiety, depression, suicidal ideation and conduct, are generated by the stress minorities suffer and the most appropriate intervention is the affirmative one. There is a high gender variance in people within the autism spectrum disorder (ASD), which is higher in girls than in boys. In 2019, Las Higueras Hospital in Talcahuano started with a trans-afirmative intervention with GI in BGA. Conclusions: It is necessary to make GI visible and depathologize it so that BGA have greater access to trans-affirmative support.
\end{abstract}

Keywords: Gender incongruity; Transgender; Boys/Girls; Adolescents, Trans-affirmative interventions.

Fecha de envío: 2020-01-22 - Fecha de aceptación: 2021-03-18

(1) Unidad de Salud Mental, Hospital las Higueras, Talcahuano, Chile.

(2) Unidad de Pediatría, Hospital las Higueras, Talcahuano, Chile.

(3) Facultad de Medicina, Universidad de Concepción, Concepción, Chile.

Autor de Correspondencia:dorca2003@hotmail.com 


\section{Introduccion}

En los países occidentales, un creciente número de adolescentes transgénero están buscando acompañamiento - acogida en clínicas especializadas en niñas, niños y adolescentes (NNA) que presentan incongruencia de género (IG) (Kaltiala-Heino et al., 2018). Además, durante la última década ha habido un importante aumento del interés por parte de la comunidad y los profesionales de la salud en temas relacionados con la población transgénero. Por su parte, los cuerpos legislativos han comenzado a evaluar los derechos de estas personas (Turban et al., 2018).

En Chile, a nivel salud se da a conocer la vía clínica para la adecuación corporal en personas con incongruencia de género (MINSAL, 2010), en donde se exponen las intervenciones de salud mental, hormonales y quirúrgicas en el manejo de personas transgénero mayores de 18 años. Al siguiente año se instruye a los establecimientos de la red asistencial con la circular 34 (MINSAL, 2011) respecto de la atención de personas trans en relación con la identificación de la persona o nombre social, registro en ficha clínica y hospitalización. En 2012 se da a conocer la circular 21 (MINSAL, 2012) que rige a la fecha y que reitera y profundiza instrucciones sobre la atención de personas trans en la red asistencial en aquellas que son de competencia en la atención ambulatorial abierta, atención primaria y de especialidades. A nivel educacional, se da a conocer la circular 0768 (MINEDUC, 2017), la cual está dirigida a todos los establecimientos educacionales del país y se enfoca en los derechos de NNA en el ámbito de la educación, particularmente en el uso del nombre social, uniforme adecuado a su identidad de género y uso de baños. A nivel legislativo, en diciembre de 2018 se publicó la ley de identidad de género 21120 (Congreso Nacional de Chile, 2018) que reconoce y da protección al derecho a la identidad de género, lo que ha permitido avanzar en visibilizar los derechos de las personas transgénero, ha incentivado la creación de programas de salud especializados en IG para NNA (Turban et al., 2018) y ha generado una derivación más expedita a estos centros de mayor especialización.

\section{Definiciones}

El género desde siempre había sido considerado binario, es decir como solamente femenino o masculino y se ha relacionado al sexo al nacer y a la orientación sexual, es decir una mujer de nacimiento debía ser de género femenino y, además al momento de su madurez sexual sentirse atraída a un hombre y por el contrario un hombre de nacimiento debía ser masculino y sentirse atraído hacia una mujer. Es necesario destacar que estos tres aspectos son diferentes entre sí y no necesariamente se van a relacionar linealmente, es decir una mujer de nacimiento se puede identificar con el género masculino y sentirse atraída sexualmente hacia una mujer. El género no es binario, ya que existen personas que se identifican con otros géneros distintos de lo femenino o masculino tales como el género fluído, agénero y género no binario.

Tabla 1: Definiciones

Sexo: condición biológica de la persona (cromosómica, hormonal, gonadal y genital).

Identidad de género: alude al autoconcepto respecto a cómo la persona siente internamente su género.

Rol de género: características consideradas como masculinas o femeninas por una cultura en particular.

Expresión de género: presentación individual de su propia identidad de género (ropa, peinados, actividades, etc.).

Orientación sexual: se refiere a quién resulta sexualmente atractivo para la persona; alude a la elección de pareja del individuo.

Incongruencia de género: discordancia entre la vivencia que la persona tiene respecto de su género y el sexo asignado al nacer.

Transgénero: la persona que vivencia una IG. Término que se utiliza indistintamente a incongruencia de género.

Cisgénero: persona cuya identidad de género es concordante con su sexo asignado al nacer. Vivencia su género como congruente.

Disforia de género: disconfort o distress al conectarse con su IG.

Condiciones asociadas: sintomatología que puede o no acompañar la IG tales como ansiedad, depresión, autoagresiones y suicidalidad.

Hombre transgénero: persona asignada a sexo femenino al nacer y que se vive hombre.

Mujer transgénero: persona asignada a sexo masculino al nacer y que se vive como mujer.

Para el propósito de esta revisión bibliográfica y el quehacer profesional en Hospital las Higueras de Talcahuano (HLH) utilizaremos la clasificación diagnóstica CIE-11 (Organización Mundial de la Salud, 2018) y los términos incongruencia de género y/o transgénero para referirnos a esta población infanto juvenil.

El objetivo de este trabajo es por una parte realizar una revisión teórica de la literatura existente en relación a la IG en NNA y además dar a conocer la realidad local en relación al tema y las intervenciones transafirmativas que se realizan por parte de los profesionales del equipo trans pediátrico en $\mathrm{HLH}$.

\section{Metodologia}

El presente trabajo corresponde a una revisión teórica y práctica. En la revisión teórica se realizó una búsqueda en PUBMED, utilizando los siguientes términos: gender incongruency in child, affirmative intervention in child and youth, autism and gender, transgender child 
and youth, gender no conforming, minority stress model, desist and persist in children. Se buscaron artículos en inglés y español, desde 2010 a 2020. De los artículos encontrados, se revisó además la bibliografía de ellos, sumando a la revisión las publicaciones que fueran pertinentes.

En la revisión práctica se realizó una sistematización de las intervenciones que se llevan a cabo a nivel endocrinológico y en salud mental, en donde cada profesional y autor aportó desde su área respectiva.

\section{Resultados}

\section{Etiología}

Se considera que la IG tiene orígenes multifactoriales, genéticos, biológicos, psicológicos y sociales. En el pasado el área de mayor interés fue psicológica y hoy el interés se ha movilizado hacia la etiología de carácter biológico (Turban et al., 2018).

Los estudios sobre la heredabilidad de la IG han reportado que los factores genéticos pueden contribuir en el desarrollo del género (Shumer et al., 2016).

Los estudios sobre el origen biológico han mostrado que las hormonas sexuales prenatales no sólo determinan el desarrollo de genitales externos e internos, sino también actúan en la diferenciación sexual del cerebro (Martinerie et al., 2018). En esta área se hace mención a la teoría neurobiológica, que se centra en el periodo de la embriogénesis y refiere que la diferenciación genital ocurre mucho antes que la sexual en el cerebro, por lo que no hay una sincronización de estos procesos y pueden no requerir similares niveles de impregnación hormonal, por lo tanto es posible que en relación a niveles variables de secreción hormonal en aquellos dos órganos, los genitales se desarrollen hacia el fenotipo mujer, mientras que el cerebro se diferencie hacia el fenotipo masculino o al contrario (Martinerie et al., 2018).

Los factores psicológicos que se han estudiado son el rol de las características de los padres en el desarrollo de la IG, tales como las expectativas, preferencias por el sexo de sus hijas(os) y la ausencia del padre; sin embargo, no hay investigaciones concluyentes al respecto (Shumer et al., 2016; Martinerie et al., 2018; Turban et al., 2018).

\section{Prevalencia}

Ha sido difícil el establecimiento de la verdadera prevalencia de la IG a causa de los cambios en la terminología y el estigma asociado a la autoidentificación (Turban et al., 2018).

En un metaanálisis de estudios de prevalencia en IG, se encontró que ha aumentado en los últimos cincuenta años llegando a 4,6 en 100000 individuos, en donde 6,8/ 100000 es para mujeres transgénero y 2,6/100000 para hombres transgénero (Arcelus et al., 2015). En 2017, en un estudio (Eisenberg et al., 2017) con un gran número de jóvenes de escuelas de Minnesota ( $\mathrm{N}: 81885)$ se publicó una prevalencia de $2,7 \%$ de jóvenes transgénero, con un $3,6 \%$ de hombres transgénero y $1,7 \%$ de mujeres transgénero.

En Alemania, en 2017 (Becker et al., 2017), se encuestó a 940 adolescentes hombres y mujeres de diez a dieciséis años y se les preguntó cuál era su experiencia actual de género (masculina o femenina) y su expresión de género y los resultados revelaron que el 4,1\% de los adolescentes eran género no binario y el 3,0\% no conformes con su género. En general se ha observado un incremento en la prevalencia debido a la despatologización y desestigmatización de la IG (Martinerie et al., 2018). Y en los adolescentes ha habido una inversión reciente en la proporción que antes favorecía a las mujeres transgénero, hoy es mayor la proporción de hombres transgénero (Littman, 2019).

En Chile existe un desconocimiento de la prevalencia de personas trans, lo que influye notablemente en las políticas públicas nacionales. La única estimación sobre diversidad sexual la ha entregado la Encuesta de Caracterización Socioeconómica Nacional CASEN (Ministerio de Desarrollo Social, 2015) y los resultados indicaron que la población que se reconoce como heterosexual es el 98 , $51 \%$, la que indica ser homosexual o lesbiana 1,04\%, bisexual 0 , $37 \%$, otra $0,02 \%$, estimándose que dentro de esta última categoría se encontraría la población transgénero. En esta misma encuesta frente a la pregunta "en cuanto a su género usted se identifica como", el 3,1\% de la población mayor de 18 años se identificó como transfemenina y el 2,3\% como transmasculino. En E.E.U.U estudios realizados estiman que $0,5 \%$ de la población se podría definir como transgénero y al hacer una extrapolación de las cifras norteamericanas a la población chilena del ultimo censo se estimaría que en Chile habrían más de 80000 personas transgénero y cada año existirían cerca de 30 nuevas personas transgénero que podrían demandar asistencia sanitaria (Zapata et al., 2019).

\section{Desarrollo de identidad de género}

Tres teorías psicológicas clásicas han elaborado explicaciones para el desarrollo de la identidad de género: la teoría psicoanalítica, la teoría del aprendizaje social y la cognitivo evolutiva, sin embargo, las tres teorías son dificilmente comparables ya que tienen planteamientos distintos (Freixas, 2012). En la teoría psicoanalítica la socialización del rol sexual se produce al superar el complejo de edipo, en la teoría del aprendizaje social los roles de género se aprenden inicialmente por observación y luego por imitación, en donde los adultos desempeñan un papel básico reforzando 
diferencialmente las conductas que les parecen apropiadas a lo femenino o masculino (Freixas, 2012), pero se ha visto que las(os) niñas(os) una vez que desarrollan su identidad de género pueden entrar en conflicto con la socialización externa (Gülgöz et al., 2019). La teoría cognitivo evolutiva desarrollada por Kohlberg (Kohlberg et al., 1967) plantea que existen estructuras centrales activas que determinan la evolución de la identidad sexual y de género y que se fundamenta en el desarrollo cognitivo del mundo social, en donde un aspecto importante es el juicio de autoclasificación que realiza la (el) niña(o) como niña o niño. Plantea tres etapas en el desarrollo, relacionadas con las etapas del desarrollo cognitivo preoperacional, operacional concreto y operacional formal: Etapa 1: adquisición de la identidad de género, surge a partir del juicio de la realidad física de que mujeres y hombres son diferentes. Etapa 2: Constancia e irreversibilidad de género, en donde se adquiere la comprensión de que el género no se puede cambiar con el tiempo y que a pesar de cambios en aspectos externos sigue estable. Etapa 3: capacidad para relativizar y criticar los contenidos sociales asignados a los roles de género.

Los teoricos del desarrollo del género actuales explican su desarrollo desde una combinación de influencias de factores biológicos (como el sexo asignado y exposición a hormonas prenatales), cognición de género y la socialización cultural e interpersonal que experimentan las niñas y niños (Gülgöz et al., 2019).

En entrevistas a madres, padres y cuidadores de jóvenes transgénero, éstos percibían las primeras señales alrededor de los cuatro años y medio, mientras las mismas niñas(os) se describían como diferentes, alrededor de los seis años (Murchison, 2016).

En un estudio norteamericano (Olson et al., 2015) se evaluó en niñas(os) de cinco a doce años la cognición de género (preferencia de género, identidad de género y preferencias explícitas de género) y los resultados mostraron que las niñas(os) transgénero no se confunden en relación a su identidad de género, piensan de acuerdo a ella y no de acuerdo a su sexo al nacer y además lo hacen desde muy pequeñas(os).

En Chile se realizó un estudio a gran escala (Organizando Trans Diversidades, 2017), mediante una encuesta online (encuesta T), en donde participaron 315 personas trans que fueron encuestadas(os) por diversos temas y entre ellos el desarrollo y específicamente a qué edad percibían que no se adecuaban al género impuesto, destacándo que la mayoría (41,3\%) lo reconoce entre los 0 y 5 años, un $39,4 \%$ lo sitúa entre los 6 y 11 años. Un 42,5\% de las(os) entrevistados declara haber reconocido su identidad de género entre los 12 y 18 años de edad, mientras que un 36,2\% lo hizo entre los 19 y 25 años de edad. Estos datos nos muestran que la mayoría se autoclasifica con un género distinto desde la primera infancia tal como fue comentado anteriormente en las etapas del desarrollo del género. También nos muestran lamentablemente el extenso tiempo que puede pasar una(o) NNA en Chile hasta que finalmente sea apoyado en su identidad y tomar contacto con un equipo de salud especializado, lo que puede conllevar dificultades de salud mental y retrasar sus intervenciones transafirmativas.

\section{Incongruencia de género y tareas del desarrollo adolescente}

Las tareas del desarrollo se refieren a los hitos normativos del desarrollo que deben alcanzarse durante una etapa del desarrollo determinada, surgen de las interacciones entre el desarrollo físico, aspectos individuales y las expectativas de la sociedad. La finalización favorable de las tareas del desarrollo de una etapa dada es un requisito previo para el éxito en las etapas posteriores. Las tareas del desarrollo de la adolescencia fueron formuladas por primera vez por Havighurst (Kaltiala-Heino et al., 2018) y comprenden aceptar el cuerpo, adoptar un rol social masculino o femenino, lograr la independencia emocional de los padres, desarrollar relaciones cercanas con los mismos géneros y opuestos, prepararse para una ocupación, prepararse para la vida familiar y la pareja, establecer un valor personal o un sistema ético y lograr un comportamiento socialmente responsable.

De todos estos aspectos, la relación con el propio cuerpo y la adquisición de un rol social de género no necesariamente binario, son desafíos de las(os) adolescentes que presentan IG (KaltialaHeino et al., 2018). Otra posible área de conflicto sería la relación cercana con pares, ya que muchas(os) de estas(os) jóvenes sufren acoso, hostigamiento o han pasado por periodos de aislamiento en la infancia y adolescencia (Kaltiala-Heino et al., 2018), por lo que desarrollar relaciones de cercanía puede ser difícil para adolescentes con IG. Con respecto a la sexualidad como uno de aquellos desafíos, se podría esperar que las(os) jóvenes transgénero presenten un desarrollo sexual tardío, debido a que es el cuerpo sexual la fuente de angustia (Kaltiala-Heino et al., 2018).

\section{Condiciones asociadas}

Las personas transgénero en general tienen más riesgo de experimentar síntomas depresivos, intentos suicidas, agresión física y psicológica (Zapata et al., 2019) y presentar ansiedad, abuso de sustancias, virus de inmunodeficiencia humana (VIH) y dificultades de acceso a la salud (Barrientos et al., 2019). Específicamente las(os) NNA transgénero muestran altas tasas de trastornos internalizantes como ansiedad y depresión (Martinerie et al., 2018), conductas de autolesión y suicidio (Shumer et al., 2016; Martinerie et al., 2018). 
En Chile, la encuesta T (Organizando Trans Diversidades, 2017) informó que un 56\% de las(os) encuestadas(os) declaró haber intentado suicidarse, siendo entre los 11 y 15 años las edades en que más se cometen los primeros intentos de suicidio (46\%) y en menor medida entre los 16 y los 18 años (26\%). Una explicación de la presencia de estas condiciones asociadas es la hipótesis del estrés de las minorías (Jaggi et al., 2018; Turban et al., 2018). Las personas transgénero o no conformes con su género están expuestas a niveles más altos de discriminación, estigmatización y violencia (Jaggi et al., 2018). El modelo de estrés de minorías se basa en la premisa de que las experiencias de estigmatización toman la forma de un llamado estrés de minoría específico que a su vez afecta el estado de salud de las personas. Los factores estresantes se dividen en distales y proximales, en donde los distales son causados por una fuente externa y los proximales se refieren a pensamientos y procesos internos subjetivos de la misma persona transgénero (Jaggi et al., 2018).

\section{Trastorno de espectro autista (TEA) e incongruencia de género}

En población transgénero existe una alta prevalencia de trastorno de espectro autista (Cooper et al., 2018; Strang et al., 2018; Turban et al., 2018; Mahfouda et al., 2019; Warrier et al., 2020), que se estima es entre el $4,8 \%$ y el $26 \%$ (Warrier et al., 2020) debido a que las personas TEA presentan altas tasas de género no conforme, siendo esto mayor en mujeres que en hombres. Las personas TEA tienen menos probabilidad de tener una sensación de afiliación a cualquier grupo de género debido a la diferencia en la cognición social y el no compartir los roles sociales (Cooper et al., 2018). Las(os) NNA género diverso y TEA son un grupo especialmente vulnerable a presentar dificultades de salud mental, particularmente del tipo internalizante (ansiedad, depresion, quejas somáticas y dificultades del pensamiento) y baja calidad de vida (Mahfouda et al., 2019). También se ha planteado una hipótesis biológica a esta alta prevalencia, la cual tiene relación con la teoría del cerebro masculino extremo (Cooper et al., 2018) y que explicaría las características de las mujeres TEA, pero no la de los hombres de esa misma condición.

En un estudio de más de 1400 adolescentes y adultos TEA, el 6,5\% de las(os) adolescentes y $11,4 \%$ de adultos reportó desear ser del otro sexo, comparado con 3\% de adolescentes y $5 \%$ de adultos controles (Van der Miesen et al., 2018). Se realizó seguimiento (Strang et al., 2018) a 22 adolescentes TEA por cerca de dos años, la mayoría de los adolescentes reportó ser género no conforme desde muy pequeño y además un tercio de los participantes dijo que otras personas habían cuestionado su diversidad de género porque eran TEA, diciéndoles que era una obsesión más que una experiencia real.
Respecto de la orientación sexual y TEA, en un gran estudio sueco (Rudolph et al., 2018) de 47000 adultos con rasgos TEA, se encontró que estas personas tenían más probabilidades que sus compañeros de describirse a sí mismos como bisexuales o como no conformes con las etiquetas de lo heterosexual, homosexual o bisexual.

\section{Incongruencia de género y familia}

El apoyo familiar es la principal herramienta para mantener la salud mental de las(os) NNA con IG. En un estudio (Olson et al., 2016) cuya muestra fueron 73 niñas y niños de entre tres y doce años que hicieron el tránsito social (cambio de nombre y apariencia) y que además eran apoyados en sus identidades por sus familias, los resultados mostraron que estas niñas y niños tenían niveles normativos de depresión y niveles de ansiedad sólo un poco más altos que la media; además de tener sintomatología internalizante notablemente más baja que los que han mostrado otros estudios con niñas y niños con IG que no han hecho el tránsito social. En Chile, la encuesta T (Organizando Trans Diversidades, 2017) informó que al interior de las familias la violencia más recurrente es el cuestionamiento de la identidad con un $97 \%$, mientras que el ignorar corresponde a un $42 \%$ y la agresión verbal a un $36 \%$. Respecto de quiénes cuestionan la identidad, un $39 \%$ señala que es su madre y un $29 \%$ que es su padre. Al comparar los datos nacionales con una de las mayores encuestas realizadas en EEUU (James et al., 2016), en donde participaron 27715 personas trans, realizada en línea y de forma anónima, observamos que la mayoría de las(os) participantes $(60 \%)$ respondieron que sus familias apoyaron su identidad trans, el $18 \%$ dijeron que no los apoyaron y el $22 \%$ dijo que sus familias no los apoyaron ni tampoco se negaron a hacerlo. Los que tuvieron el apoyo de sus familias directas tenían menos probabilidades de registrar una variedad de experiencias negativas relacionadas con la estabilidad económica y de la salud como estar sin hogar, intentar suicidarse o un malestar psicológico grave. Los datos nos muestran que el apoyo del núcleo familiar directo es muy relevante para una persona trans y específicamente para las(os) NNA, ya que aún son dependientes económica, psicológica y afectivamente. Nos queda mucho por hacer como país en garantizar un mayor apoyo familiar a las identidades trans.

\section{Persistencia y desistencia}

Las familias de NNA habitualmente preguntan a los profesionales, si es que la IG persistirá o desistirá más adelante en el tiempo. Autores como Temple Newhook (Steensma \& Cohen-Kettenis, 2018) reportan la desistencia de la IG en un $80 \%$, sin embargo, Steensma (Steensma \& Cohen-Kettenis, 2018) afirma que estos datos estarían basados en estudios con problemas metodológicos respecto de los conceptos de IG y basados también en la comparación de estudios con muestras distintas. 
En las niñas y niños la intensidad de la incongruencia parece ser un predictor de la persistencia (Steensma et al., 2013) y el rango de edad de diez a trece parece ser crucial para la persistencia o desistencia (Steensma et al., 2011) ya que en este periodo comienzan a emerger las características sexuales secundarias del sexo no deseado, aumentando la disforia o la angustia frente a los cambios corporales, surgiendo así como un indicador de persistencia en su identidad de género (Shumer et al., 2016).

Otros aspectos importantes a observar en las niñas y niños que persisten en su IG es la insistencia, persistencia y consistencia en su identidad de género y las declaraciones de las niñas(os) respecto de su identidad de género (Yo soy un niño) v/s su expresión de género (me gustaría ser un niño). (Murchison, 2016).

Hoy en día muchas madres y padres en foros online describen que sus hijas(os) han tenido un inicio rápido de identificarse como transgénero, al igual que el grupo de pares a quienes sus hijas(os) frecuentan y antes de revelarlo a su familia pasan bastante tiempo en uso de internet y redes sociales. Este nuevo grupo de inicio rápido (rapid onset of gender dysphoria ROGD) ha sido estudiado (Littman, 2019) en forma descriptiva por medio de 256 reportes de madres y padres, quienes informaron que sus hijas(os) en su mayoría eran mujeres asignadas al nacer (82\%), con una edad promedio de 15,2 años al momento que se identificaron como transgénero y el $41 \%$ había expresado una orientación sexual no heterosexual antes de identificarse como transgénero. Un $86,7 \%$ de los padres informó que junto al inicio rápido de la incongruencia de género también observaron que sus hijas(os) tuvieron un aumento en el uso de redes sociales e internet y pertenecieron a un grupo de amigas(os) en el que uno o varias(os) también se identificaron como transgénero.

Al hacer un análisis de esta investigación es necesario decir que si bien es cierto muchas(os) NNA se identifican como transgénero en la adolescencia, tal como lo de muestra la encuesta $T$, en donde un $15,6 \%$ se da cuenta que no se adecua al género impuesto entre los 12 a 18 años. Otras(os) NNA perciben desde pequeñas(os) que no se adecúan a su género, pero aún no toman contacto con un concepto que las(os) identifique, por lo que muchas(os) se identifican durante mucho tiempo como homosexual, lesbiana o bisexual hasta que finalmente por medio de redes sociales e internet toman contacto con el concepto transgénero y se identifican con él, es así que las madres y padres de la investigación de Littman reportan que un $54,2 \%$ pedían consejo para saber si eran o no transgénero. Muchas(os) NNA recurren a internet y redes sociales para conocerse e identificarse a través de otras(os).

Una limitación que muestra la investigación de Litman es que obtiene información desde las madres y padres, quienes a veces niegan o no quieren reconocer las primeras manifestaciones de incongruencia de género en la infancia de sus hijas(os), es así como un $76,5 \%$ de los padres consideraron que sus hijas(os) estaban equivocadas(os) en su creencia de ser transgénero. Es importante saber que muchas(os) NNA pueden iniciar rápidamente la identificación transgénero, pero hay que tomar con cautela esta información y se require más investigación al respecto.

\section{Intervenciones transafirmativas}

Las intervenciones se basan en el modelo afirmativo, el cual considera que todos los resultados de la identidad de género son igualmente válidos y deseables y permite a las niñas(os) que expresen su deseo de transición social, hacerlo después de un asesoramiento cuidadoso (Turban et al., 2018).

Las intervenciones médicas se clasifican en reversibles, parcialmente reversibles e irreversibles. Dentro de las reversibles se encuentra el bloqueo puberal y es la primera intervención que se puede realizar desde el estadio 2 de Tanner usando análogos de la hormona liberadora de gonadotropina. Luego alrededor de los catorce o dieciséis años las(os) adolescentes pueden optar a la siguiente intervención que es la terapia hormonal cruzada con estrógenos o testosterona (Hembree et al., 2017). A la edad legal para la adultez las(os) usuarias(os) pueden someterse a intervenciones quirúrgicas como se encuentra sugerido en la vía clinica (MINSAL, 2010).

\section{Intervenciones transafirmativas con NNA y sus familias en Hospital las Higueras de Talcahuano (HLH)}

Desde el mes de abril de 2019, en el HLH y con un equipo multidisciplinario, se formalizó la atención a NNA transgénero, en donde el enfoque de trabajo se basa en el modelo de intervención transafirmativo. Con el objetivo de despatologizar la intervención de NNA, el ingreso al programa se realiza desde endocrinología (ver figura 1), luego se realiza interconsulta a psiquiatría y finalmente a psicología. El objetivo antes mencionado va en directa relación con las necesidades sentidas por la comunidad trans de despatologización - buen trato y diálogo - participación social, las cuales fueron determinadas en una investigación cualitativa en la mesa trans compuesta por personas de la comunidad trans, del Servicio de Salud Talcahuano y del Hospital Las Higueras de Talcahuano (Valenzuela et al., 2020).

A la fecha el equipo atiende 19 usuarias(os), las(os) cuales son atendidas(os) en salud mental infanto juvenil hasta los 18 años y en endocrinología infantil hasta los 15 años, luego de esa edad son derivadas(os) a la continuación de sus intervenciones con el equipo adulto. Del total de usuarias(os) atendidas(os), 18 son hombres trans $(89,4 \%)$ y 2 son mujeres trans $(10,5 \%)$, siendo estos datos locales coincidentes con el aumento de la prevalencia del hombre trans que 
se ha venido señalando en distintas publicaciones internacionales (Zucker, 2017; Litman, 2019). Del total de usuarias(os), 2 son TEA trans (10,5\%), porcentaje que se ajusta a la prevalencia observada internacionalmente (Warrier et al., 2020) y de ellas(os) 1 es mujer TEA - trans (5,2\%) y 1 hombre TEA - trans (5,2\%). Respecto de los grupos etáreos, el mayor grupo de usuarias(os) es el adolescente $(94,7 \%)$ y en menor medida el grupo preadolescente (5,2\%). Dentro de la adolescencia el 42,1\% corresponde a adolescents de 16 años, luego el $21,0 \%$ corresponde a adolescents de 18 años y $15,7 \%$ corresponde a adolescents de 15 años.

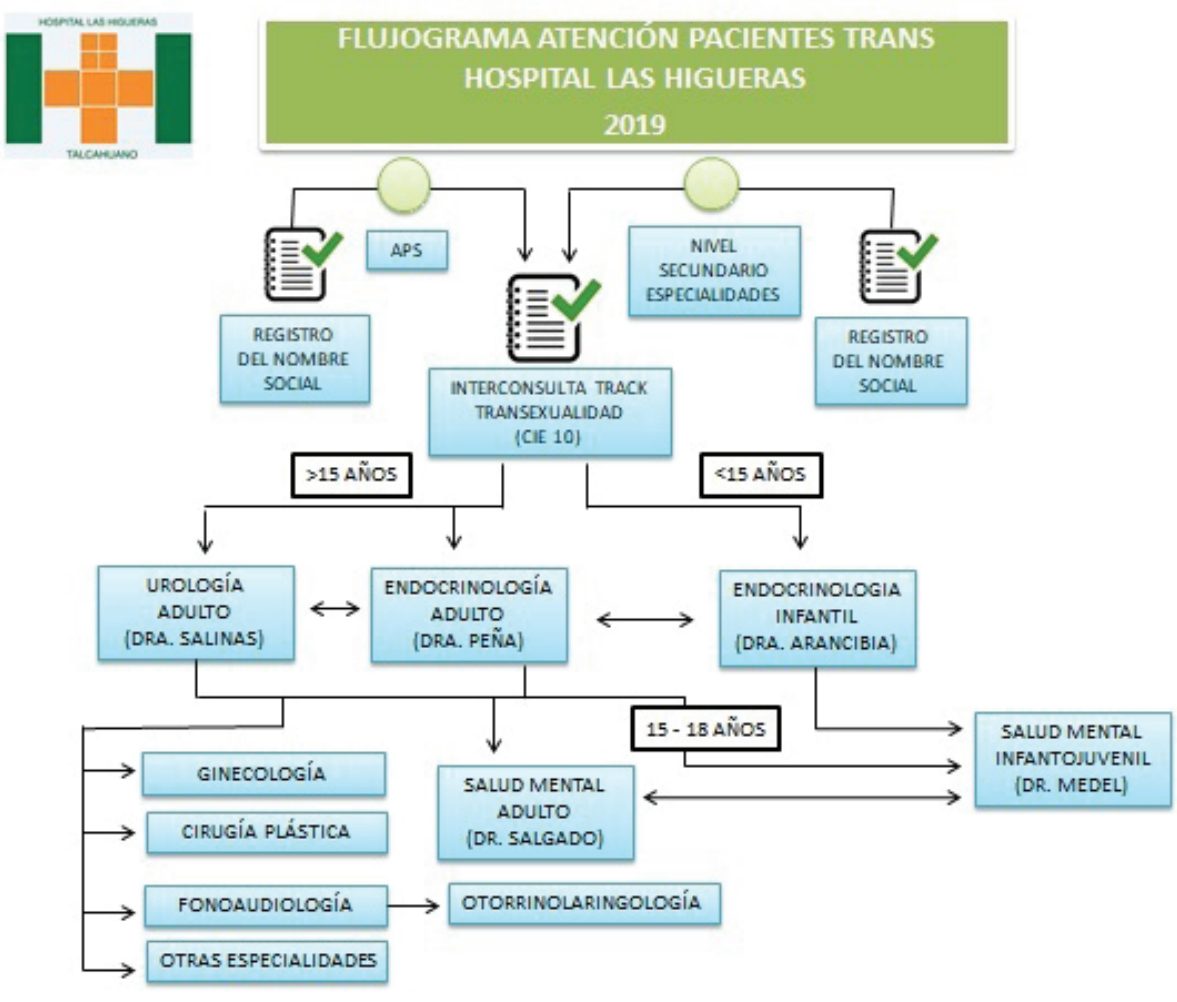

Figura 1: Flujograma de atención a usuarias(os) transgénero en Hospital las Higueras.

\section{Intervenciones en endocrinología:}

Etapa prepuberal: se debe realizar evaluación diferencial con variaciones del desarrollo sexual y vigilancia activa frente a la aparición de los primeros signos de pubertad (estadio 2 de Tanner) (Devoto et al., 2016). La aparición de botón mamario en niñas regularmente ocurre entre los ocho y doce años y el crecimiento testicular mayor a 4 cc en niños generalmente es entre los nueve y los trece años. La transición social es una intervención que se puede realizar en esta etapa y es completamente reversible asociada con disminución de depresión y ansiedad en niñas(os) prepúberes transgénero (Scherer, 2016).

Etapa puberal: Al pesquisar estadio 2 de Tanner se indica frenación del eje hipotálamo hipófisis gónada para evitar progresión de pubertad. Antes de iniciar este tratamiento se debe cumplir con los siguientes criterios de elegibilidad para uso de análogo del GnRH (Hembree et al., 2017):

\section{Cumplir con criterios de IG}

2. Estadio 2 de Tanner

3. Ausencia de alteraciones psiquiátricas

4. Estar en algún tratamiento que no contraindique la readecuación corporal

5. Evaluación psicológica y apoyo social adecuado, comprensión realista de resultados esperados, conocimiento de riesgos y beneficios de esta terapia reversible (Devoto et al., 2016). 
A la edad de dieciséis años (Hembree et al., 2017) se recomienda iniciar la terapia hormonal cruzada para inducir pubertad acorde a su identidad de género.

Criterios de elegibilidad para uso de terapia hormonal cruzada (Hembree et al., 2017):

\section{Persistencia de IG}

2. Que las condiciones asociadas psicológicas, médicas o sociales estén abordadas y resueltas.

3. Adolescente con capacidad mental suficiente (dieciséis años) para entender las consecuencias, riesgos y beneficios de esta terapia parcialmente irreversible, además de dar su asentimiento y consentimiento informado.

\section{Actividades en endocrinología}

A continuación, se detallan las actividades que se realizan en esta área con NNA y sus familias:

Tabla 2: Actividades Endocrinología

Anamnesis, examen físico.

Discusión de las metas deseadas y posibles del tratamiento con los padres y/o cuidadores y las(os) NNA.

Discusión de efectos adversos con las madres, padres y/o cuidadores de NNA.

Derivación para consejería en fertilidad.

Evaluación de riesgos.

Interconsulta a otras especialidades en caso de detección de riesgos específicos.

Solicitud de exámenes de laboratorio e imágenes.

Consentimiento y asentimiento informado para padres y NNA.

Inicio de supresión puberal.

Inicio de inducción puberal

Seguimiento.

Reunión clínica para estudio de casos.

\section{Intervenciones en salud mental:}

Se realizan en primera instancia dos entrevistas de evaluación con psiquiatra infanto juvenil. Mediante estas entrevistas se establece la presencia de IG, condiciones asociadas y se descarta trastorno psiquiátrico. Se realiza derivación a psicóloga y luego que la psicóloga realiza dos sesiones de evaluación, se analiza caso a caso y se realiza informe por parte de psiquiatra que confirma la IG y sugiere iniciar intervención con endocrinóloga y de salud mental.

\section{Actividades en salud mental}

Tabla 3: Actividades Salud Mental

Coordinación en colegios

Educación en colegios en el tema Trans

Intervención mensual grupal con familia

Intervención grupal con los NNA

Visitas domiciliarias

Acompañamiento y asesoramiento en aspectos legales en relación al cambio de nombre social en ficha, registral en Tribunales de Familia o registro civil de acuerdo a la edad, circular 0768 MINEDUC y derivación de caso a OPD en caso de vulneración de derechos.

Reunión clínica

\section{Discusion y conclusiones}

La IG en NNA se ha ido visibilizando cada vez más, debido de la despatologización en las clasificaciones diagnósticas, a cambios progresivos en la sociedad al ir entendiendo el género como un concepto no binario, al desarrollo de cuerpos legislativos que reconocen y protegen la identidad de género y al esfuerzo diario que realizan las organizaciones que apoyan a la población LGBTQ+.

Este trabajo al visibilizar la IG, creemos que va en la dirección de aportar en disminuir el estrés de minorías, el estigma y la discriminación, que de acuerdo a la literatura genera sintomatología ansiosa, depresiva, bajo autoconcepto y suicidalidad.

Todos los ambientes y contextos donde se desenvuelven y desarrollan las(os) NNA con IG juegan un rol determinante en disminuir la estigmatización y/o patologización de la condición, es así como las instituciones educativas y también las de salud, deben crear conciencia de esta realidad y adecuar su funcionamiento para disminuir el surgimiento de sintomatología disfórica.

Respecto de la prevalencia, en el equipo trans pediátrico también nos hemos ido encontrando con un mayor número de usuarios NNA hombres transgénero $(89,5 \%)$ que ingresan al programa en búsqueda de intervenciones, por que lo advertimos una disminución de la prevalencia de mujeres transgénero adolescentes, lo que coincide con la literatura internacional revisada y que por lo demás requiere futuras investigaciones para ir comprendiendo estos cambios.

Por su parte la familia realiza un gran aporte en la reducción de síntomas al respaldar y apoyar en el cambio social a NNA, por lo que los esfuerzos de los profesionales también deben estar encaminados a conseguir el apoyo necesario desde los padres, madres 
y cuidadores. Respecto de las intervenciones a nivel familiar otorgadas por el equipo Higueras, se ha valorado bastante el grupo para familias, ya que allí pueden compartir experiencias con otras familias que se encuentran en el mismo proceso.

Con las(os) adolescentes, se ha valorado la intervención grupal debido al espacio que brinda el grupo de compartir experiencias con otras(os) que se encuentran en una misma situación, no sentirse sola(o) y ayudar en tareas del desarrollo adolescente.

Otra población que es necesario conocer y apoyar son las(os) NNA TEA con IG, que por lo demás están en un mayor riesgo de desarrollar condiciones asociadas por pertenecer a dos grupos de minorías.

La creación de un equipo especializado en la atención precoz e integral de NNA con IG en el Hospital las Higueras, es una experiencia pionera en la región del Bío Bío, pero quedan muchos desafíos futuros en relación a la temática, como la formación de más equipos especializados en intervención trans pediátrico a nivel país, la creación de una red en el tema a nivel infanto juvenil, establecer mayor conección con educación y con las organizaciones de la comunidad. Esperamos ir acercándonos a estos desafíos en pro del bienestar de las(os) NNA con IG.

\section{Contribuciones y reconocimientos:}

No existe conflicto de intereses de participación como activistas de organizaciones LGBTIQ+, tampoco el trabajo recibió financiamiento.

\section{Referencias}

Arcelus J, Bouman W, Van Den Noortgate W, Claes L, Witcomb G. \& Fernandez-Aranda F. (2015). Systematic review and meta-analysis of prevalence studies in transsexualism. European Psychiatry 30, 807-815.

Barrientos J, Saiz J, Gómez F, Guzmán-González M, Espinoza-Tapia

R, Cárdenas M. \& Bahamondes J. (2019). Psykhe 28.

Becker L, Ravens-Sieberer U, Ottová-Jordan V. \& Schulte-Markwort M. (2017). Prevalence of adolescent gender experiences and gender expression in germany. Journal Adolescent Health 61, 83-90.

Congreso Nacional de Chile. (2018). Ley de Identidad de Género 21120. Accedido en http://www.congreso.cl/ el 25 de noviembre de 2019.

Cooper K, Smith L. \& Russell A. (2018). Gender identity in autism: sex differences in social affiliation with gender groups. Journal of Autism and Developmental Disorders 48, 3995-4006.

Devoto E, Ríos \& Aravena L. Trastorno de identidad de género. (2016). Parte II: terapia endocrinológica en el proceso de readecuación corporal. Revista Chilena de Endocrinología y Diabetes 9, 58-64.
Eisenberg M, Gower A, McMorris B, Rider G, Shea G \& Coleman E. (2017). Risk and protective in the lives of transgender/gender nonconforming adolescents. Journal Adolescent Health 61, 521-526.

Freixas A. (2012). La adquisición del género: el lugar de la educación en el desarrollo de la identidad sexual. Apuntes de Psicología 30, 155-164.

Gülgöz S, Glazier J, Enright E, Alonso D, Durwood L, Fast A, Lowe R, Ji CH, Heer J, Martin C. \& Olson K. (2019). Similarity in transgender and cisgender children's gender development. Proceedings of the National Academy of Sciences 116.

Hembree W, Cohen-Kettenis P, Gooren L, Hannema S, Meyer W, Murad M, Rosenthal S, Safer J, Tangpricha V. \& TSjoen G. (2017). Endocrine treatment of gender-dysphoric/gender-incongruent persons: an endocrine society clinical practice guideline. Journal of Clininical Endocrinology and Metabolism $102,1-35$.

Jaggi T, Jellestad L, Corbisiero S, Schaefer D, Jenewein J, Schneeberger A, Kuhn A. \& García D. (2018). Gender minority stress and depressive symptoms in transitioned swiss transpersons. Journal Biomedicine and Biotechnology (4): 1-10.

James S, Herman J, Rankin S, Keisling M, Mottet L. \& Anafi M. (2016). The report of the 2015 U.S. Transgender Survey. Washington, DC: National Center for Transgender Equality. Accedido en: https://www. transequality.org/sites/default/files/docs/USTS-Full-Report-FINAL. PDF el 9 de febrero de 2021.

Kaltiala-Heino R, Bergman H, Tyolajavi M. \& Frisen L. (2018). Gender dysphoria in adolescence: current perspective. Adolescent Health, Medicine and Therapeutics 9, 31-41.

Kohlberg L \& Zigler E. (1967). The impact of cognitive madurity on the development of sex-role attitudes in the years 4 to 8 . Genetic Psychology Monographs 67, 89-165.

Littman L. (2019). Parent reports of adolescents and young adults perceived to show signs of a rapid onset of gender dysphoria. PLOS ONE 14.

Mahfouda S, Panos Ch, Whitehouse A, Thomas C, Maybery M, Strauss P, Zepf F, O'Donovan A, Van Hall H, Saunders L, Moore J. \& Lin A. (2019). Mental health correlates of autism spectrum disorder in gender diverse young people: evidence from a specialized child and adolescent gender clinic in Australia. Journal of Clinical Medicine 8, 1503. 
Martinerie L, Condat A, Bargiacchi A, Bremont-Weill C, De Vries A. \& Hannema S. (2018). Approach to the management of children and adolescents with gender dysphoria. European Journal of Endocrinology 179, 219-237.

MINEDUC (2017). Circular 0768. MAT: Derechos de niños, niñas y estudiantes trans en el ámbito de la educación. Accedida en: https://www.supereduc.cl/wp-content/uploads/2017/04/ORD-N\%C2\%BA0768-DERECHOS-DE-NI\%C3\%91AS-NI\%C3\%91OS-Y-ESTUDIANTES-TRANS-EN-EL-\%C3\%81MBITO-DE-LA-EDUCACI\%C3\%93N-A-SOSTENEDORES.pdf. el 1 de febrero de 2021.

Ministerio de Desarrollo Social (2015). Encuesta de caracterización socioeconómica nacional. Accedido en: http://www.desarrollosocialyfamilia.gob.cl/pdf/upload/IDS_INAL_FCM_3.pdf. El 2 de febrero de 2021.

MINSAL (2010). Vía clínica para la adecuación corporal en personas con incongruencia entre sexo físico e identidad de género. Accedido en: https://www.minsal.cl/portal/url/item/d126e58ba4cb53f5e040010165017912.pdf. El 2 de febrero de 2021.

MINSAL (2011). Circular 34. Accedida en: http://www.movilh.cl/ documentacion/trans/Circular-Salud-Trans.pdf. El 2 de febrero de 2021.

MINSAL (2012) Circular 21. Accedida en: http://diprece.minsal.cl/ wrdprss_minsal/wp-content/uploads/2015/01/CIRCULAR-21-Reitera-Atenci\%C3\%B3n-de-personas-trans.pdf. El 3 de febrero de 2021.

Murchison G. (2016). Supporting and caring for transgender children. American Academy of Pediatrics. Sept.

Olson K, Key A \& Eaton N. (2015). Gender cognition in transgender children. Psychological Science 26, 467-474.

Olson K, Durwood L, DeMeules M \& McLaughlin K. (2016). Mental health of transgender children who are supported in their identities. Pediatrics 137.

Organización Mundial de la Salud. (2018). Clasificación internacional de enfermedades CIE-11. Accedido en: https://icd.who.int/es. El 20 de noviembre de 2019.

Organizando Trans Diversidades (2017). Informe ejecutivo Encuesta T. Santiago, Chile: Autor. Extraído de encuesta-t.cl. Accedido en: https://otdchile.org/biblioteca/encuesta-t-2/. El 4 de febrero de 2021. Rudolph C, Lundin A, Ahs J, Dalmac C \& Kosidou K. (2018). Brief report: sexual orientation in individuals with autistic traits: population based study of 47000 adults in stockholm county. Journal of Autism Developmental Disorders 48, 619-624.
Scherer I. (2016). Social transition: supporting our youngest transgender children. Pediatrics 137.

Shumer D, Nokoff N \& Spack N. (2016). Advances in the care of transgender children and adolescents. Advances in Pediatrics 63, 79-102.

Steensma T, Biemond R, De Boer F \& Cohen-Kettenis P. (2011). Desisting and persisting gender dysphoria after childhood: a qualitative follow-up study. Clinical Child Psychology and Psychiatry16, 499-516.

Steensma T, McGuire J, Kreukets B, Beekman A. \& Cohen-Kettenis P. (2013). Factors associated with desistence and persistence of childhood gender dysphoria: a quantitative follow up study. Journal of the American Academy of Child and Adolescent Psychiatry 52, 582-590.

Steensma T \& Cohen-Kettenis P. (2018). A critical commentary on "a critical commentary on follow-up studies and "desistence" theories about transgender and gender non-conforming children". International Journal of Transgender 19, 225-230.

Strang J, Meagher H, Kenworthy L, De Vries A, Menvielle E, Leibowit S, Janssen A, Cohen-Kettenis P, Shumer D, Edwards-Leeper L, Pleak R, Spack N, Karasic D, Schreier H, Balleur A, Tishelman A, Ehrensaft D, Rodnan L, Kuschner E, Mandel F, Caretto A, Lewis H. \& Anthony L. (2018). Initial clinical guidelines for co-occurring autism spectrum disorder and gender dysphoria or incongruence in adolescents. Journal of Clinical Child and Adolescent Psychology 47, 105-115.

Strang J, Powers M, Knauss M, Sibarium E, Leibowit S, Kenworthy L, Sadikova E, Wyss S, Willing L, Caplan R, Pervez N, Nowak J, Gohari D, Gomez-Lobo V, Call D. \& Anthony L. (2018). "They thought it was an obsession": trajectories and perspectives of autistic transgender and gender-diverse adolescents. Journal of Autism Developmental Disorders 48, 4039-4055.

Turban J, De Vries A, Zucker K. \& Shandianloo S. (2018). Transgénero y Jóvenes no conformes con su género. Manual de Salud Mental Infantily Adolescente de la IACAPAP. Ginebra: Asociación Internacional de Psiquiatría del Niño y el Adolescente y Profesiones Afines. Cap. 3 Ed. Irarrázabal M, Martin A. trad. Ortega, Espinoza M, Valenzuela A $\&$ Cartes R. (2020). Salud comunitaria, la experiencia de salud trans en el Servicio de Salud Talcahuano. Psicoperspectivas. Individuo y Sociedad 19.

Van der Miesen A, Hurley H, Bal A. \& De Vries A. (2018). Prevalence of the wish to be of the opposite gender in adolescents and adults with autism spectrum disorder. Archives of Sexual Behavior 47, 2307-2317. 
Retamal et al.

Warrier V, Greenberg D, Weir E, Buckingham C, Smith P, Lai M, Allison C. \& Baron - Cohen S. (2020). Elevated rates of autism, other neurodevelopmental and psychiatric diagnoses, and autistic traits in transgender and gender-diverse individuals. Nature Communications 11, 3959.
Zapata A, Diaz K, Barra L, Maureira L, Linares J. \& Zapata F. (2019). Atención de salud de personas transgéneros para medicos no especialistas en Chile. Revista Médica de Chile 147. 